\title{
STUDY OF DYNAMIC GAS RESPONSE OF MICROWAVE PLASMA ENHANCED CHEMICAL VAPOR DEPOSITION SYSTEMS BY OPTICAL EMISSION SPECTROSCOPY
}

\author{
1,2Nicolas LAMBERT, ${ }^{1,3}$ Vincent MORTET \\ ${ }^{1} \mathrm{FZU}$ - Institute of Physics of the Czech Academy of Sciences, Prague, Czech Republic, EU, mortetv@fzu.cz \\ ${ }^{2}$ Faculty of Electrical Engineering, Czech Technical University in Prague, Prague, Czech Republic, EU, \\ lambert@fzu.cz \\ ${ }^{3}$ Faculty of Biomedical Engineering, Czech Technical University in Prague, Kladno, Czech Republic, EU, \\ mortetv@fzu.cz
}

https://doi.org/10.37904/nanocon.2021.4379

\begin{abstract}
Microwave Plasma Enhanced Chemical Vapor Deposition (MWPECVD) is widely used for the growth and the study of synthetic doped diamond and its electronic and electrochemical applications. Recent results show the possible enhancement of dopant incorporation in diamond using pulsed gas injection in the reactor. Therefore, understanding the gas dynamic of the MWPECVD reactors is crucial to the optimization of the gas flows and diamond doping. In this work, we present a method to determine the gas dynamic response of a MWPECVD reactor. We determined the dynamic response of injected gas in a NIRIM type and a linear antenna type MWPECVD reactor by recording light emission from a hydrogen plasma during the instantaneous injection of a small fraction of nitrogen using either Optical Emission Spectroscopy (OES) or the combination of a photodiode and an optical band filter. Using linear system theory, we developed a program to determine the dynamic response of the reactor to an arbitrary shaped gas flow input from its pulse response.
\end{abstract}

Keywords: Pulse response, MWPECVD reactor, OES, gas dynamics, linear system theory

\section{INTRODUCTION}

Microwave Plasma Enhanced Chemical Vapor Deposition (MWPECVD) is widely used for the growth synthetic doped diamond used in electronic and electrochemical applications. Recent results show the possible enhancement of dopant incorporation using pulsed gas injection [1]. Therefore, understanding the gas dynamic of the MWPECVD reactors is crucial to the optimization of the gas flows and diamond doping.

In this work, we present and compare two methods for measuring the time response of two different MWPECVD reactors, with different volumes and different operating pressures, to a short $\mathrm{N}_{2}$ gas pulse: a NIRIM type system [2] and a linear antenna MWPECVD system [3]. The time response is obtained by monitoring the intensity of the emission line at $337 \mathrm{~nm}$ attributed $\mathrm{N}_{2}$ [4] in a hydrogen plasma by recording the voltage across a photodiode combined to a filter with a bandwidth centered around $340 \mathrm{~nm}$ and by Optical Emission Spectroscopy (OES) using a spectrometer with a wavelength range from 200 to $1100 \mathrm{~nm}$. Both methods are compared and the advantages and flaws of each are discussed. Data were fitted using the impulse response equation proposed by $\mathrm{H}$. Pendar et al. [5]. Finally, using linear system theory, it was possible to predict the dynamic response of the reactor to an arbitrary shaped gas flow input from its pulse response [6,7].

\section{EXPERIMENTAL SETUP}

The conditions of gas flow, pressure, and the volume of both the NIRIM and linear antenna MWPECVD system are summarized in Table 1. 
Table 1 Plasma conditions

\begin{tabular}{|c|c|c|c|}
\hline Reactor & $\mathbf{H}_{\mathbf{2}}$ gas flow $(\mathbf{s c c m})$ & Pressure (mbar) & Chamber volume (L) \\
\hline NIRIM & 200 & 70 & 1.4 \\
\hline Linear antenna MWPECVD & 200 & 0.42 & 160 \\
\hline
\end{tabular}

The measurement setup is described in Figure 1. $\mathrm{H}_{2}$ flow was controlled by a MKS mass flow controller. In order to generate the $\mathrm{N}_{2}$ gas pulse in the plasma a small volume of $\mathrm{N}_{2}$ (ca. $1 \mathrm{ml}$ ) was trapped between two ball valves at a pressure of 2.6 bar and then released in the chamber.

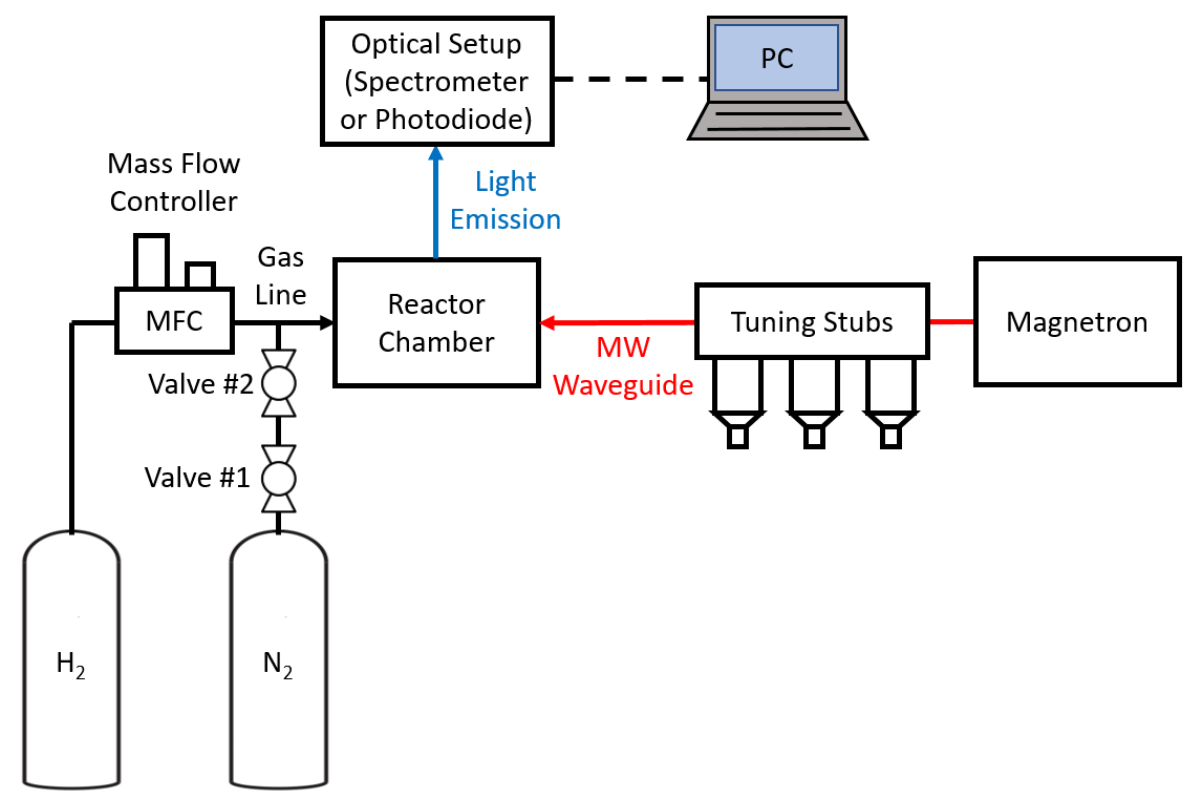

Figure 1 Measurement setup

To measure the time response of the reactors to the $\mathrm{N}_{2}$ gas pulse, the light emission from the plasma was monitored using the following setups. In the first setup, the emission line at $337 \mathrm{~nm}$ attributed to molecular $\mathrm{N}_{2}$ was monitored using a Thorlabs SM05PD3A photodiode with a wavelength range from 320 to $1100 \mathrm{~nm}$ in combination with a Thorlabs FB340-10 band pass filter centered at $340 \mathrm{~nm}$ with a full width half maximum of $10 \mathrm{~nm}$. The photodiode voltage was recorded as a function of time using a Keysight 34461A multimeter and saved on a computer using Keysight BenchVue software. A homemade brass holder was designed to combine the photodiode and filter in a closed package and isolate it from any parasitic light sources. The main advantages of this setup are its simplicity and its operation speed with a sampling rate of about $20 \mathrm{~Hz}$. However, its major flaw is its poor selectivity as it is impossible to measure and remove possible variation of the continuous background light coming from the hydrogen plasma during the gas pulse, which can overshadow the variation of the measured emission line. In the second setup, we used a Stellarnet BLACKComet-SR spectrometer, which can record spectra from 200 to $1100 \mathrm{~nm}$. An integration time of $25 \mathrm{~ms}$ was used to retain a high sampling rate comparable to the first setup. The acquired spectra were analyzed using an algorithm in Origin $\AA$ software to remove the variation of the continuous background from the $\mathrm{N}_{2}$ emission peak at $337 \mathrm{~nm}$.

\section{EXPERIMENTAL RESULTS}

The experimental dynamic responses of both reactors to a $\mathrm{N}_{2}$ pulse measured with the photodiode and the spectrometer are presented in Figure 2. The time responses recorded on the MWPECVD linear antenna system have a very similar shape contrary to the ones recorded on the NIRIM reactor. The signal measured 
in the NIRIM reactor using the photodiode decreases below its reference value after the signal reaches its maximum. However, since the data extracted from the second measurement setup do not exhibit this unrealistic variation, we conclude that this anomaly is due to a change in the background signal during the pulse.
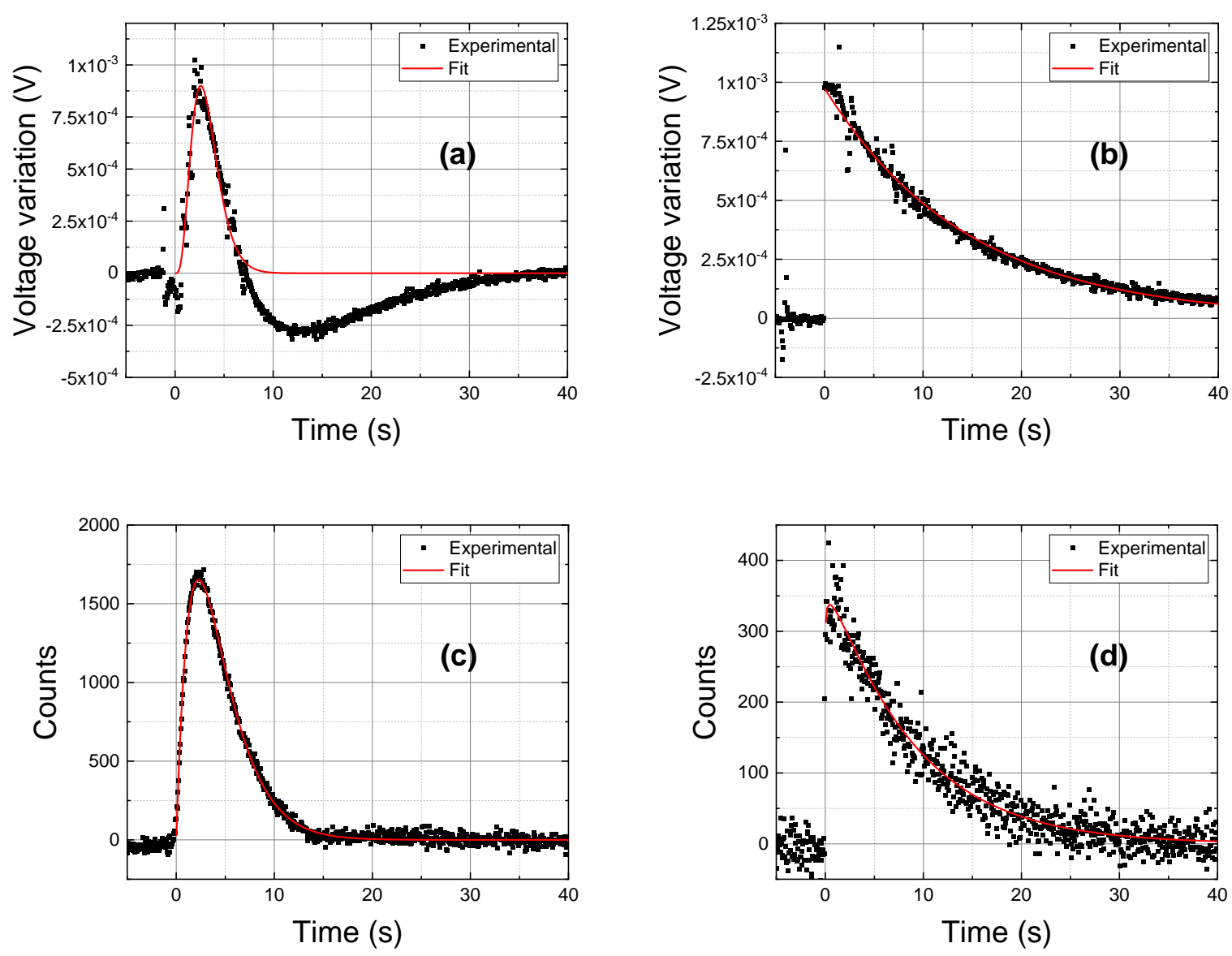

Figure 2 Experimental dynamic pulse responses and fitting curves measured on (a) the NIRIM reactor and (b) the MWPECVD linear antenna reactor using the photodiode and experimental dynamic pulse responses and fitting curves measured on (c) the NIRIM reactor and (d) the MWPECVD linear antenna reactor using the spectrometer

The time responses were fitted using the equation proposed by $\mathrm{H}$. Pendar et al. [5]:

$f(t)=\alpha t^{m} e^{-\beta t}$

where $t$ is the time and $\alpha, m$, and $\beta$ are fitting parameters. $\beta$ is characteristic of the exponential decay of the signal which dominates for large value of time while $m$ characterizes the rising portion of the signal for small value of time. The curve reaches its maximum for $t=\frac{m}{\beta}$ with a value of:

$f\left(\frac{m}{\beta}\right)=\alpha\left(\frac{m}{\beta}\right)^{m} e^{-m}$

Equation (1) fits well the experimental data as shown in Figure 2 except in Figure 2a, which corresponds to the measurement carried out with the photodiode on the NIRIM reactor due to the variation of the background intensity, which cannot be taken into account in the model. The fitting parameters for each condition are presented in Table 2. Despite the differences in shape of the recorded signals on the NIRIM system, the time 
responses reach their maximum at a similar time of $t=\frac{m}{\beta}=2.7 \mathrm{~s}$ (photodiode) and $t=\frac{m}{\beta}=2.2 \mathrm{~s}$ (spectrometer). In the case of the MWPECVD linear antenna system, a larger value of $\beta$ was obtained using the spectrometer compared to the photodiode, which reflects the faster decay of the signal when the time response is measured using the oscilloscope. This difference can be attributed to a change in the background, which affects the signal recorded with the photodiode similarly to what was already observed on the NIRIM system. The position of the maximum calculated from the fitting parameters is $t=\frac{m}{\beta}=2.3 \times 10^{-7} \mathrm{~s}$ (photodiode) and $t=\frac{m}{\beta}=4.6 \times 10^{-1} \mathrm{~s}$ (spectrometer). Even though the results largely differ, this difference is attributed to the limited time resolution of the measurement methods.

The measurements done with the photodiode are less noisy than the ones obtained using the spectrometer. It is particularly visible in Figure $\mathbf{2 b}$ and Figure $\mathbf{2 d}$. However, the photodiode is unfortunately sensitive to background changes during the measurement as observed in Figure 2a. For this reason, the setup using the photodiode is less reliable for measuring the time response of the reactors in comparison to the one using the spectrometer, which should be preferred.

The fitting parameters for the two reactors determined from the spectrometer setup data analysis, show a much faster rising part (i.e. a smaller $\frac{m}{\beta}$ value) and a slower decay (i.e. a larger $\beta$ value) for the linear antenna MWPECVD reactor compared to the NIRIM reactor. Those results are attributed to the different volumes of the two reactors [5].

Table 2 Fitting parameters

\begin{tabular}{|c|c|c|c|}
\hline Measurement Method & Reactor & $\boldsymbol{\beta}\left(\mathbf{s}^{-1}\right)$ & $\boldsymbol{m}$ \\
\hline \multirow{2}{*}{ Photodiode } & NIRIM & 1.5 & 4.0 \\
\cline { 2 - 4 } & Linear antenna & $6.9 \times 10^{-2}$ & $1.6 \times 10^{-8}$ \\
\hline \multirow{2}{*}{ Spectrometer } & NIRIM & $4.5 \times 10^{-1}$ & 1.0 \\
\cline { 2 - 4 } & Linear antenna & $1.2 \times 10^{-1}$ & $5.5 \times 10^{-2}$ \\
\hline
\end{tabular}

Using linear system theory, it is possible to determine the time response of the reactors to an arbitrary shaped gas flow input from its pulse response [6,7]. The dynamic response is given by equation (3):

$c(t)=\int_{0}^{t} u(\tau) h(t-\tau) d \tau$

Where $u(t)$ is an arbitrary input signal and $h(t)$ is the impulse time response of the reactor.

In order to test this model, we have compared the experimental time response of the NIRIM reactor to a oneminute-long, square shaped flow of $\mathrm{N}_{2}$ with an amplitude of $2 \mathrm{sccm}$ injected into the hydrogen plasma using a MKS mass flow controller in the same conditions as described in Table 1, and its modeled response. The experimental response was recorded using the spectrometer and the calculated response was obtained using equations (1) and (3) and the fitting parameters in Table 2. The curves presented in Figure 3 compare the experimental results to the calculated responses using both the photodiode or the spectrometer fitting parameters. It is clearly visible that the modeled response using the spectrometer fitting parameters more closely matches the experimental data. The discrepancy observed in the rising part of the curve is attributed to the slow response time of the mass flow controller $(500 \mathrm{~ms})$, which is smaller than the rising time of the NIRIM reactor dynamic response of $2.7 \mathrm{~s}$, but not negligible. 


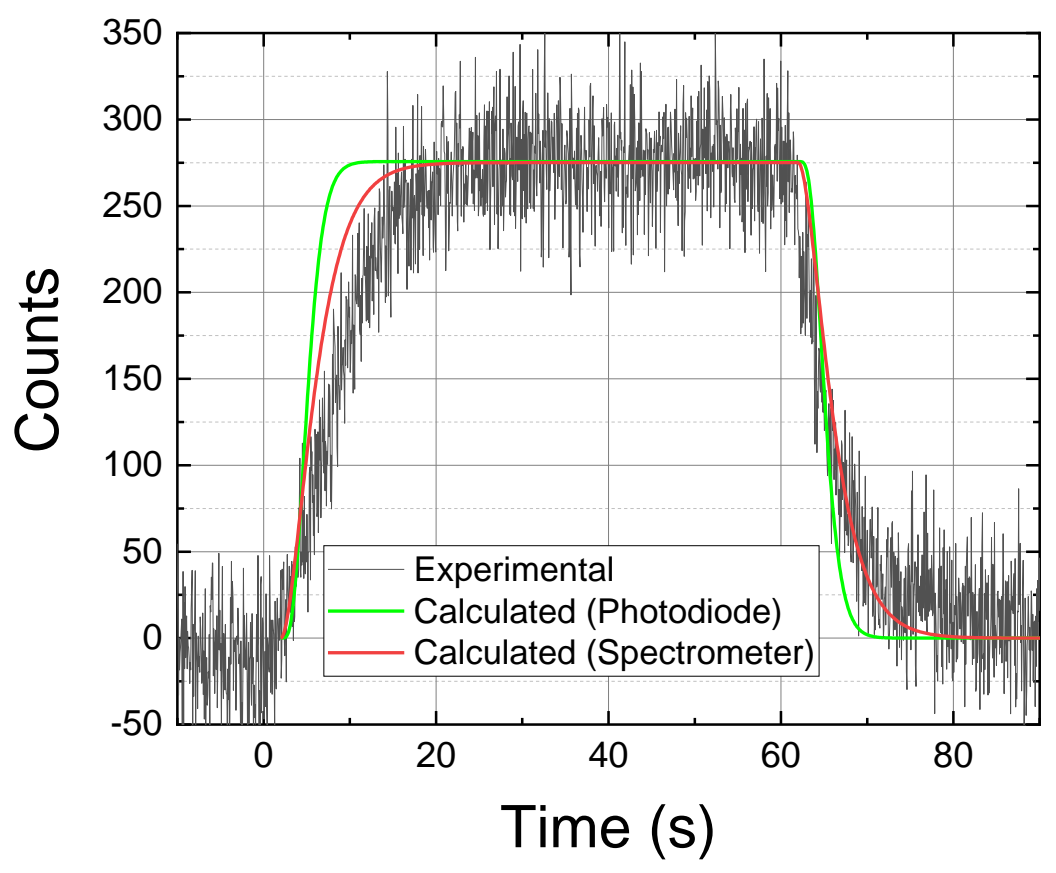

Figure 3 Recorded and simulated response of the NIRIM reactor to a one-minute-long, square shaped flow of $\mathrm{N}_{2}$ with an amplitude of $2 \mathrm{sccm}$ using the plasma conditions described in Table 1

\section{CONCLUSION}

Two different measurement setups were used to measure the time response of a NIRIM reactor and a linear antenna MWPECVD reactor to a $\mathrm{N}_{2}$ gas pulse: one simple setup based on the voltage measurement across a photodiode combined with an optical filter, and another using a compact spectrometer. Despite a better signal to noise ratio compared to the spectrometer, the photodiode has the disadvantage of being sensitive to background changes during the experiment, which ultimately makes it unsuitable for measurement of the dynamic response of MWPECVD reactors. The time response depends on the physical characteristic of the reactor and operating pressure: a faster rising time and a slower decay were observed for the linear antenna MWPECVD reactor compared to the NIRIM system. Those differences can be explained by the large difference in volume between the two reactor chambers. Using linear system theory and the fitting curve of the time response obtained with the spectrometer, it was also possible to successfully predict the response of the NIRIM system to a one-minute-long square $\mathrm{N}_{2}$ pulse.

\section{ACKNOWLEDGEMENTS}

The financial support from the following sources is gratefully acknowledged: The Czech Science Foundation grant ID: GACR 21-03538S, the SGS Student Grant ID: SGS21/057/OHK3/1T/13, and the Operational Program Research, Development, and Education financed by European Structural and Investment Funds and the Czech Ministry of Education, Youth and Sports (Project No. SOLID21 CZ.02.1.01/0.0/0.0/16_019/0000760).

\section{REFERENCES}

[1] LAMBERT, N., WEISS, Z., KLIMŠA, L., FEKETE, L. and MORTET, V. Highly phosphorus-doped nanocrystalline diamond growth and properties. In: 31st International Conference on Diamond and Carbon Materials. Sep. 2021. 
[2] KAMO, M., SATO, Y., MATSUMOTO, S. and SETAKA, N. Diamond synthesis from gas phase in microwave plasma. Journal of Crystal Growth. [online]. 1983, vol. 62, no. 3, pp. 642-644. Available from: https://doi.org/10.1016/0022-0248(83)90411-6.

[3] FENDRYCH F. et al. Growth and characterization of nanodiamond layers prepared using the plasma-enhanced linear antennas microwave CVD system. J. Phys. D: Appl. Phys. [online]. 2010, vol. 43, no. 37, p. 374018. Available from: https://doi.org/10.1088/0022-3727/43/37/374018.

[4] QAYYUM, A., ZEB, S., NAVEED, M. A., REHMAN, N. U., GHAURI, S. A. and ZAKAULLAH, M. Optical emission spectroscopy of Ar-N2 mixture plasma. Journal of Quantitative Spectroscopy and Radiative Transfer. [online]. 2007, vol. 107, no. 3, pp. 361-371. Available from: https://doi.org/10.1016/j.jqsrt.2007.02.008.

[5] PENDAR H. and SOCHA, J. J. Estimation of Instantaneous Gas Exchange in Flow-Through Respirometry Systems: A Modern Revision of Bartholomew's Z-Transform Method. PLOS ONE. 2015, vol. 10, no. 10, p. e0139508. Available from: https://doi.org/10.1371/journal.pone.0139508.

[6] PORAT, B. A course in digital signal processing. New York: John Wiley, 1997.

[7] HAMILTON, J. D. Time series analysis. Princeton, N.J: Princeton University Press, 1994. 